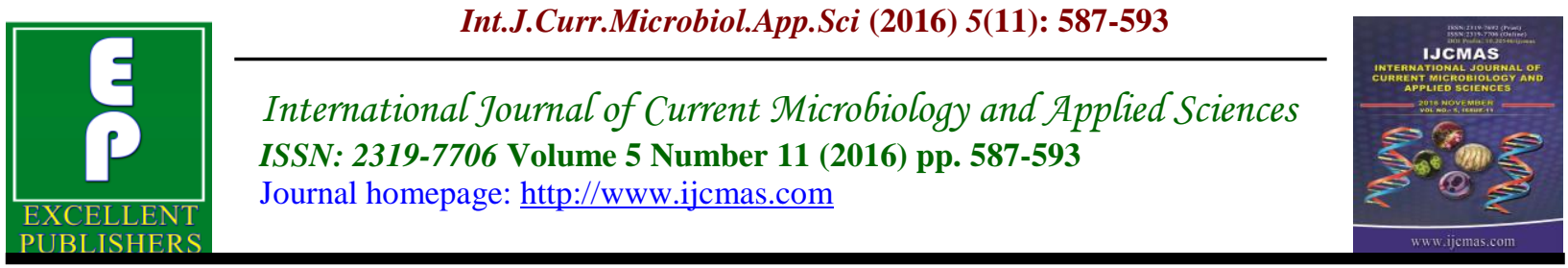

Original Research Article

http://dx.doi.org/10.20546/ijcmas.2016.511.069

\title{
Clinico-Mycologicalal Profile of Dermatophyte Species in a Tertiary Care Center
}

\author{
Nabila Akbar $^{1^{*}}$ and M. Kalyani ${ }^{2}$ \\ Saveetha Medical College and hospital, Thandalam, Kanchipuram Dist., India \\ *Corresponding author
}

Keywords

Dermatophyte,

Tinea,

Trichophyton

rubrum

$\mathrm{KOH}$.

Article Info

Accepted:

26 October 2016

Available Online:

10 November 2016

\section{A B S T R A C T}

Dermatophytosis is one of the most common causes of superficial fungal infections. These organisms colonize the keratin tissues and are restricted to the nonliving cornified layer of the epidermis. This study aims at detection of the rate of incidences of dermatophytic infections (group wise) and etiological agents. To find the Prevalence of dermatophytosis in out patients attending a tertiary care center. Samples from 114 cases of clinically suspected patients of dermatophytosis were collected over a period of six months. Detailed history was taken and thorough clinical examination was done, following which samples were collected and subjected to $\mathrm{KOH}$ test and culture using SDA and DTM. Of the 114 samples collected from patients, we found that dermatophytoses is more common in males $75(65.7 \%)$ than females $39(34.2 \%)$. The commonest clinical diagnosis was $T$. corporis (47\%), least common being T. unguim 5\%. Of 114 sample, $\mathrm{KOH}$ positivity was $68(59.6 \%)$ and culture positive was found to be $43(37.7 \%)$. Out of 43 samples Trichophyton 32(74.4\%), Microsporum 7(16.27) and 4 (9.3\%) Epidermophyton were the dermatophytes isolated. Trichophyton rubrum was found to be the commonest infecting species in this study. Young males were more affected and T. corporis is the commonest clinical diagnosis. Dermatophytosis can be prevented by a meticulous personal hygiene. Proper education and personal hygiene will definitely help the population to prevent contracting the infection and in active disease to approach for treatment.

\section{Introduction}

Dermatophytosis are the most common types of cutaneous fungal infections seen in man affecting skin, hair, and nails, in both developed and developing countries. Dermatophytosis or ringworm infection is a very common superficial fungal infection in hot and humid climate in the tropical and subtropical countries like India (Singh et al., 2003). Millions of people worldwide are affected by Superficial fungal infections (SFIs), with an estimated lifetime risk of
10\%-20\% (Sahin et al., 2004). The WHO estimates global prevalence of dermatophytosis to be approaching $20 \%$.They are the most frequently encountered causative agents of SFIs, leading to tinea infections, which are generally classified according to the body site affected.

Commonly called Tinea, dermatophytosis are one of the most prevalent superficial 
fungal infections due to crowded living and poor hygiene in developing countries (Mehrotra et al., 1978). The incidence of dermatophytosis has also increased over the years owing to factors like immunosuppression, antibiotic abuse, corticosteroids, antineoplastic drugs and animal contact. This type of infection involves only the keratinized layers like hair, skin and nails due to their ability to utilize keratin as a nutrient source, i.e. they have a unique enzymatic capacity [keratinase].

Dermatophytes are molds that can invade the stratum corneum of the skin or other keratinized tissues derived from epidermis, such as hair and nails. It may cause infections (dermatophytosis) at most skin sites, although the feet, groin, scalp, and nails are most commonly affected (Mandell).

The present study was undertaken with a view to find out the clinical pattern of Dermatophytes and to identify the commonest distribution of Dermatophytes.

\section{Materials and Methods}

This study was carried out for a period of six months between May 2015- November 2015 in a tertiary care center in Chennai. The sample size was 114. Samples from all clinically suspected cases of Dermatophyte infection were collected. The affected area was first cleaned with $70 \%$ alcohol to remove surface contamination and after the alcohol dries the various samples collected were skin scraping, hair plucking and nail clippings.

Samples were collected from at least two sites. Samples from patients of any ages and both sexes were included. The samples were rejected if topical steroids were applied or anti-fungal therapy was started. Skin scraping was done using a sterile surgical blade and collected in sterile black envelope, hair plucking was done with the help of sterile epilator and nail clippings were also collected. A part of the samples was first subjected to $\mathrm{KOH}$ mount. $10 \% \mathrm{KOH}$ was used for skin scraping, $20 \%$ for hair plucking and $40 \% \mathrm{KOH}$ used for nail samples. The other portion was used for culture on SDA (Saburoads Dextrose Agar) as well as on DTM (Dermatophyte test medium). A positive culture was further subjected to slide culture and urease test (where applicable) for identification.

\section{Results and Discussion}

During this six months study, 114 samples were collected from suspected cases of Dermatophyte infection, attending the Dermatology Department.

Of the 114 samples collected from patients we found that dermatophytoses is more common in males 75 (65.7\%) (Females 39 $\{34.2 \%\})$. The commonest age group affected in both the sexes was between 2130 years. Age and sex distribution is shown in table 1

Among 114 patients $75 \%$ of the cases were factory workers of lower socioeconomic status $\mathrm{KOH}$ positivity and culture positivity was $68(59.6 \%)$ and $43(35.9 \%)$ respectively. As shown in figure 1 and 2

Of the 43 culture positive samples, 6 were from patients suffering from diabetes mellitus. Out of 114 samples the clinical diagnosis was as follows. The commonest clinical diagnosis was $T$. corporis noted as $47 \%$, followed by T.cruris $23 \%$, T. pedis 13\%, T. capitis 5\%, T. barbae 5\%, T. faciei $2 \%$ and T. unguium 5\%. As shown in Table 2. Culture positivity in 114 samples was 
found to be $43(37.7 \%)$. Out of which the genus isolated were Trichophyton 32(74.4\%), Microsporum 7(16.27) and only $4(9.3 \%)$ isolates of Epidermophyton as shown in table 3 and fig 3,4.

\section{Clinico-Mycological correlation of dermatophyte species}

Our study showed T.rubrum was isolated in 21 cases, 11of the isolates were T.mentagrophytes, 4 of the isolates were E.flocossum and 7 of M.gypseum were also isolated. The culture positivity as per the clinical diagnosis was as follows, $T$. corporis $20(46.5 \%)$ followed by T.cruris 12(27.9\%), T.pedis 2(4.6\%), T.capitis 2(4.6\%), T.barbae 1(2.3\%), T.faciei 2(4.6\%) and T. unguim 3(6.9\%) as shown in Table 4

The distribution of Dermatophytes depends on the geographical area and course of time. (5). In the present study of six months duration, 114 samples was collected from patients (i.e.) clinically suspected cases of dermatophytosis attending Department of Dermatology in a tertiary care center.

Among 114 samples collected from patients, we found that dermatophytosis is more common in males than in females (i.e.) males $75(65.7 \%)$ and females $39(34.2 \%)$ similar to a study by Surendran et al., (Surendran et al., 2014) Their study revealed that dermatophytosis was more in males $(62 \%)$ than in females $(38 \%)$ and also a study by Narasimhalu et al., showed that on gender based prevalence males (59\%) were found to be more commonly affected, which may be attributed to the increased outdoor physical activities and increased sweating. Also the infection is commonly seen in the age group of 21-30 in both the sexes. Like seen in an article by Surendran et al., showing the commonest age group were between 16-30 years followed by 3145 years, another study by Vikesh Kumar Bhatia et al., it was observed that $64.9 \%$ patients fell in the age group of $20-50$ years while $28.3 \%$ and $6.8 \%$ patients were in the age group of 1-20 years and $>50$ years respectively.

In our study we find that the $\mathrm{KOH}$ positivity was more than the culture positivity (i.e.) $\mathrm{KOH}$ and Culture positivity was $68(59.6 \%)$ and $41(35.9 \%)$ respectively. These findings highlight the importance of both direct microscopy and culture in definitive diagnosis of fungal infection. A study by Sahai et al., (2011) also shows the $\mathrm{KOH}$ positivity to be more than the culture positivity i.e. out of 165 cases enrolled, 148 $(89.6 \%)$ cases were positive by direct microscopy and 137 (83\%) cases were culture positive. The $\mathrm{KOH}$ positivity and culture negativity in the current study could be related to the fact that some patients could have had recurrence of the infection with prior antifungal therapy.

Table.1 Correlation of gender and age in patients

\begin{tabular}{|l|l|l|l|l|l|l|l|}
\hline & $\mathbf{0 - 1 0}$ & $\mathbf{1 1 - 2 0}$ & $\mathbf{2 1 - 3 0}$ & $\mathbf{3 1 - 4 0}$ & $\mathbf{4 1 - 5 0}$ & $\mathbf{5 0}$ & Total \\
\hline Male & 3 & 4 & 37 & 16 & 9 & 6 & 75 \\
\hline Female & 2 & 2 & 16 & 12 & 5 & 2 & 39 \\
\hline Total & 5 & 6 & 53 & 28 & 14 & 8 & 114 \\
\hline
\end{tabular}


Table.2 Sex distribution of different clinical types of dermatophytes

\begin{tabular}{|c|l|l|l|l|}
\hline Clinical types & Male & Female & Total & Percentage \\
\hline$T$. corporis & 35 & 18 & 53 & $46.4 \%$ \\
\hline$T$. cruris & 16 & 7 & 23 & $20.1 \%$ \\
\hline$T$. pedis & 4 & 6 & 10 & $8.7 \%$ \\
\hline$T$. capitis & 3 & 2 & 5 & $4.3 \%$ \\
\hline$T$. barbae & 9 & 0 & 9 & $7.8 \%$ \\
\hline$T$. faciei & 4 & 3 & 7 & $6.1 \%$ \\
\hline$T$. unguium & 4 & 3 & 7 & $6.1 \%$ \\
\hline Total & 75 & 39 & 114 & $100 \%$ \\
\hline
\end{tabular}

Table.3 Species distribution of dermatophytes

\begin{tabular}{|l|l|c|c|}
\hline S.No & Isolates & No of Isolates & Percentage \\
\hline $\mathbf{1}$ & T.rubrum & 21 & $48.8 \%$ \\
\hline $\mathbf{2}$ & T.mentagrophytes & 11 & $25.5 \%$ \\
\hline $\mathbf{3}$ & E.floccosum & 4 & $9.3 \%$ \\
\hline $\mathbf{4}$ & M.gypseum & 7 & $16.2 \%$ \\
\hline
\end{tabular}

Table.4 Clinico -Mycological correlation of dermatophyte species isolated

\begin{tabular}{|c|c|c|c|c|c|c|c|c|c|c|}
\hline \multirow{3}{*}{$\begin{array}{l}\text { Clinical } \\
\text { diagnosis }\end{array}$} & \multicolumn{6}{|c|}{ Dermatophytes } & & & \multirow{2}{*}{\multicolumn{2}{|c|}{$\frac{\text { Total }}{(43)}$}} \\
\hline & \multicolumn{2}{|c|}{$\begin{array}{l}\text { T.rubrum } \\
(21)\end{array}$} & \multicolumn{2}{|c|}{$\begin{array}{l}\text { T.mentagro } \\
\text { phytes }(11)\end{array}$} & \multicolumn{2}{|c|}{$\begin{array}{l}\text { M.gypseum } \\
\text { (7) }\end{array}$} & \multicolumn{2}{|c|}{$\begin{array}{l}\text { E.flocossum } \\
\text { (4) }\end{array}$} & & \\
\hline & $\mathrm{NO}$ & $\%$ & $\mathrm{NO}$ & $\%$ & $\mathrm{NO}$ & $\%$ & $\mathrm{NO}$ & $\%$ & $\mathrm{NO}$ & $\%$ \\
\hline T. corporis & 8 & $38.1 \%$ & 5 & $45.4 \%$ & 7 & $100 \%$ & & $0 \%$ & 20 & $46.5 \%$ \\
\hline T. cruris & 6 & $28.5 \%$ & 4 & $36.3 \%$ & - & $\%$ & 2 & $50 \%$ & 12 & $27.9 \%$ \\
\hline T.pedis & 1 & $4.7 \%$ & - & - & & $\%$ & 1 & $25 \%$ & 2 & $4.6 \%$ \\
\hline T. capitis & 2 & $9.5 \%$ & & & - & - & - & - & 2 & $4.6 \%$ \\
\hline T. barbae & 1 & $4.7 \%$ & 1 & $9.0 \%$ & - & - & - & - & 2 & $2.3 \%$ \\
\hline T. faciei & & & 1 & $9.0 \%$ & - & - & 1 & $25 \%$ & 2 & $4.6 \%$ \\
\hline T. unguim & 3 & $14.2 \%$ & - & - & - & - & - & - & 3 & $6.9 \%$ \\
\hline
\end{tabular}

Fig.1 KOH positivity of samples

$40.4 \%$

$59.6 \%$ 
Fig.2 Culture positivity of samples

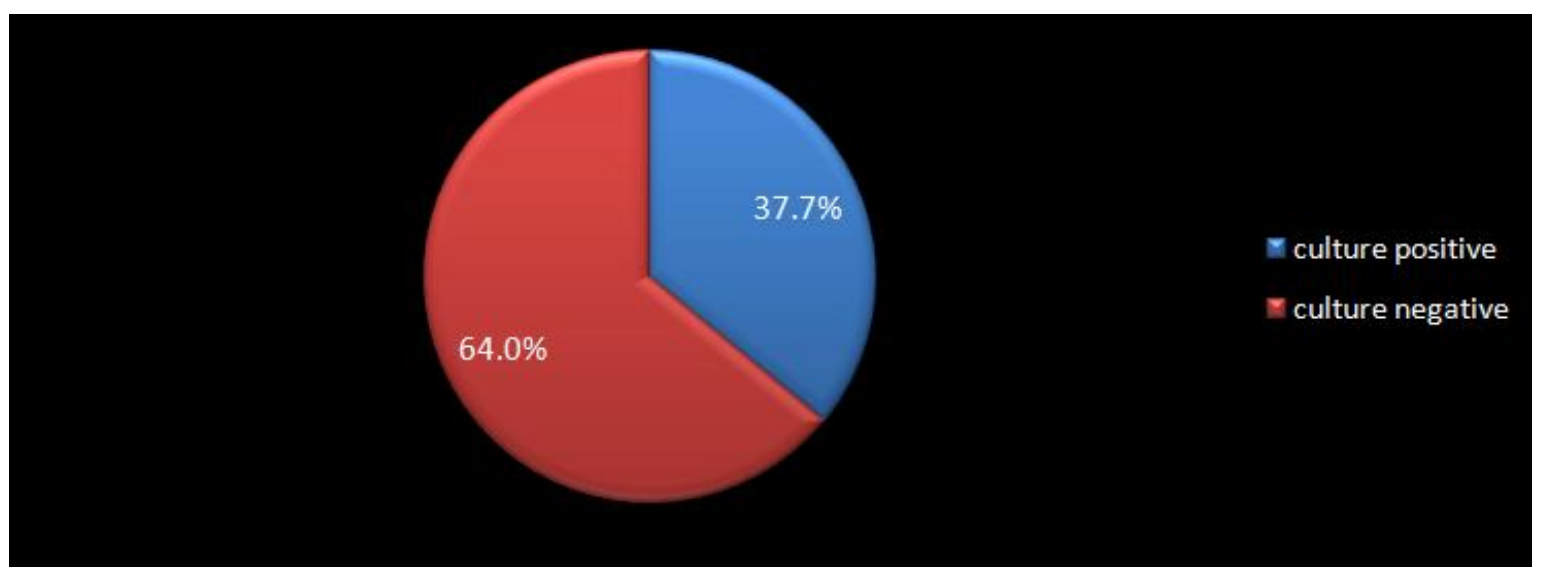

Fig.3 Distribution of dermatophytes

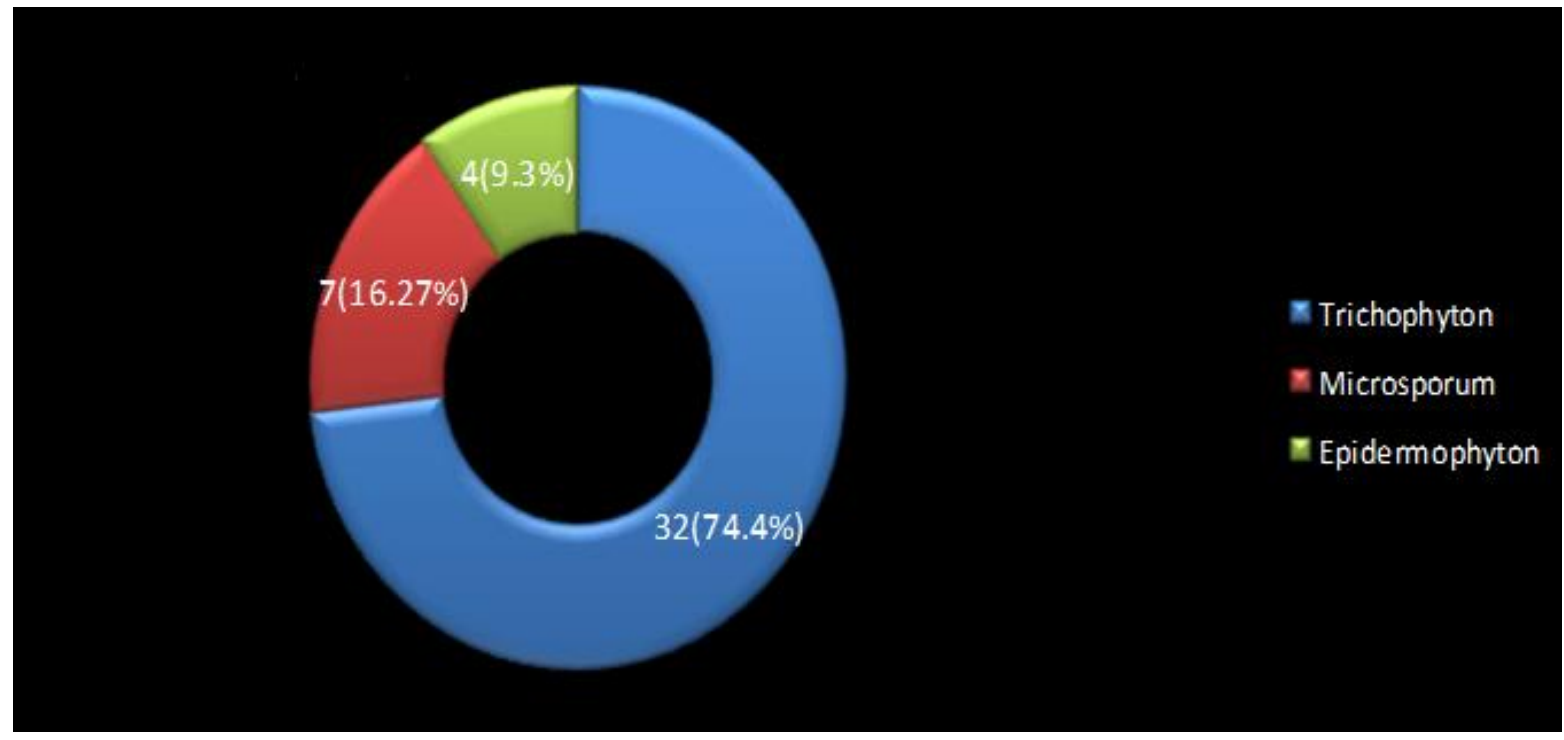

Fig.4 Species distribution of dermatophytes.

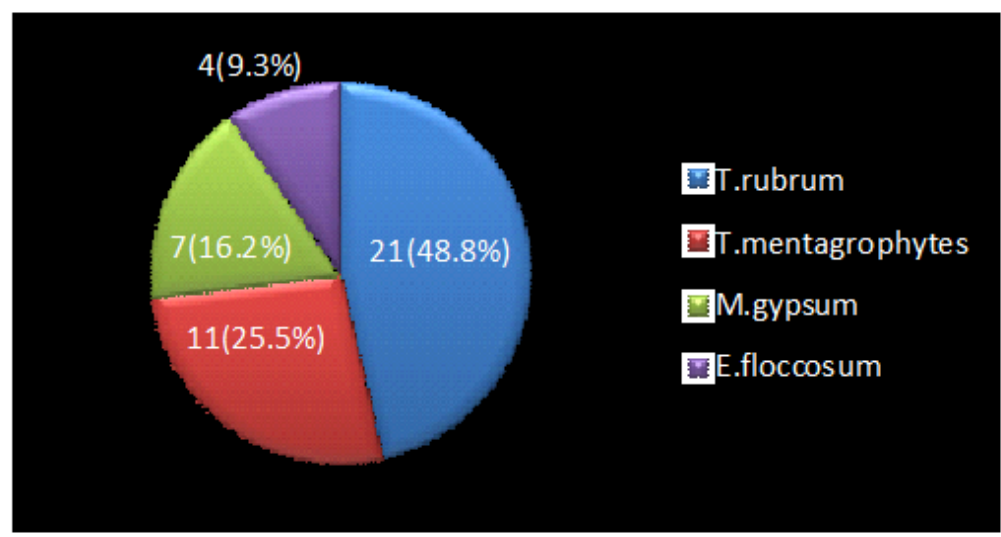


Fig.5 Colony morphology on DTM

\section{1) E.Flocossum}

2) T.mentagrophytes

3) M.gypseum

4) T.rubrum

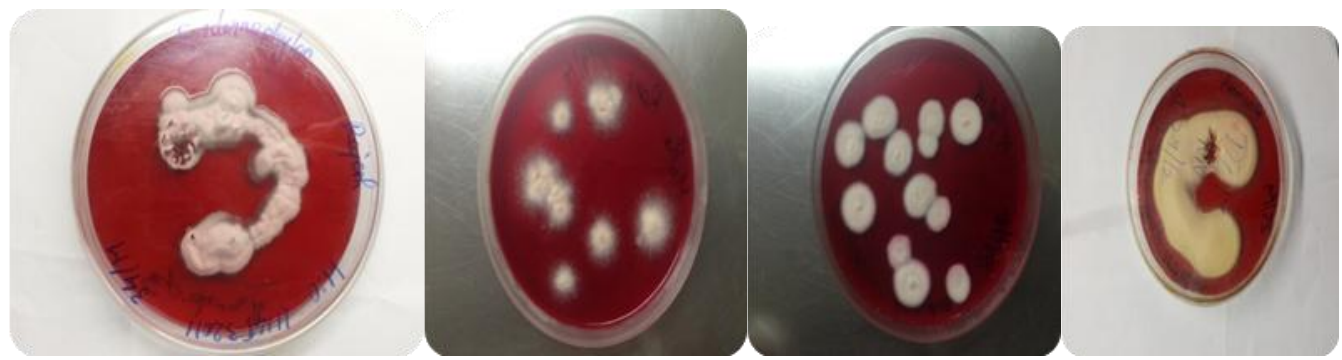

5) Sabouraud dextrose agar



It is notable that of the 43 culture positive samples 6 where from patients suffering from diabetes mellitus. In this study we found that out of 114 samples the clinical diagnosis was as follows. The commonest clinical diagnosis was $T$. corporis noted as $47 \%$, followed by $T$. cruris $23 \%, T$. pedis 13, T. capitis 5\%, T. barbae 5\%, T. faciei $2 \%$ and $T$. unguium $5 \%$.

A study by Kennedy Kumar et $a l^{(10)}$ shows similar findings $T$. corporis as $70 \%$ followed by $T$. cruris $18 \%, T$. pedis $4 \%, T$. capitis $1 \%, T$. barbae $1 \%, T$. faciei $3 \%$ and $T$. mannum 3\%. Further the findings of the present study were comparable with other studies from different places of India Singh et al., (2003); Sen et al., (2006) where the most common clinical type of dermatophytosis observed in the present study was Tinea corporis (48\%) followed by Tinea cruris (19\%) and Tinea unguium (11\%).

Culture positive samples were 43(37.7\%) among 114 samples. In our study the most common isolate was Trichophyton
32(74.4\%), followed by Microsporum 7(16.27) and 4 (9.3\%) Epidermophyton which is similar to Lakshmanan et al., (2015) who found that, Trichophyton rubrum was the commonest (79\%), followed by T.mentagrophytes (14.5\%), Microsporum canis $(3.2 \%)$ and $M$. gypseum (3.2\%). In a study by Patel et al., (2010) from Gujarat, dermatophytes commonly isolated were $T$. rubrum, followed by $T$ mentagrophytes, $M$. canis, and $M$. gypseum. In the findings of the study by Muhsin et al., (1999) from Iraq shows Epidermophyton floccosum $(31.4 \%)$ was the commonest Dermatophyte isolated.

In conclusion, $\mathrm{KOH}$ and culture positivity from a fungal sample rests heavily on the method of sample collection. Gender wise and age wise distribution suggests male preponderance and majority of the people within the 21 to 30 years age group, which reflects the external heat and humidity they are exposed to in their work environment. Personal history, occupational history and clinical examination aid in diagnosis. Tinea 
corporis was the most common clinical presentation. The commonest species of Dermatophyte isolated was T.rubrum.This study signifies the importance of proper hygiene which will prevent spread of the infection.

\section{References}

Kennedy Kumar, Anupma Jyoti Kindo, J., Kalyani, S., Anandan. 2007. Clinico mycological profile of dermatophytic skin infections in a tertiary care center - a cross sectional study -Sri Ramachandra J. Med., Vol. 1 Issue 2.

Lakshmanan, A., P. Ganeshkumar, S. Raam Mohan, M. Hemamalini, R. Madhavan. 2015. Epidemiological and clinical pattern of dermatomycoses in rural IndiaEmployee State Insurance Corporation Medical College and Post-graduate Institute of Medical Science and Research, Chennai, IndiaIJMM, Volume : 33 | Issue : 5.

Mandell, Dauglus Bennett's. Principle and Practice of Infectious Diseases, Sixth Edition, Volume 2, Chapter 265, Dermatophytosis and Other SuperficiDl Mycoses: 3051-3062.

Mehrotra, H.K., Bajaj, A.K., Gupta, S.C., Mehrotra, T.N., Atal, P.R., et al. 1978. A study of dermatophytes at Allahabad. Indian J. Pathol. Microbiol., 21: 131-139.

Muhsin, T.M., al-Rubaiy, K.K., al-Duboon, A.H. 1999. Characteristics of dermatophytoses in Basrah, Iraq. Mycoses, 42: 335-8.

Narasimhalu, C.R.V., M. Kalyani, Somendar, S. A Cross-Sectional, Clinico-Mycological Research Study of Prevalence, Aetiology, Speciation and Sensitivity of Superficial Fungal Infection in Indian Patients. $J$.
Clin. Exp. Dermatol. Res., 7: 324. doi: 10.4172/2155-9554.1000324 Page

Patel, P., Mulla, S., Patel, D., Shrimali, G. 2010. A Study of Superficial Mycosis in South Gujarat Region. Natl. J. Community Med., 1: 85-8.

Sahai, S., Mishra, D. 2011. Change in spectrum of dermatophytes isolated from superficial mycoses cases: First report from Central India. Indian J. Dermatol. Venereol. Leprol., 77: 335-6.

Sahin, I., Oksuz, S., Kaya, D., Sencan, I., Cetinkaya, R. 2004. Dermatophytes in the rural area of Duzce, Turkey. Mycoses, 47: 470-474.

Seebacher, C., Bouchara, J.P., Mignon, B. 2008. Updates on the epidemiology of dermatophyte infections. Mycopathologia, 166: 335-52.

Sen, S.S., Rasul, E.S. 2006. Dermatophytes in Assam, Indian J. Med. Microbiol., 24: 774.

Singh, S., Beena, P.M. 2003. Comparative study of different microscopic technique and culture media for isolation of dermatophytes. Indian J. Med. Microbiol., 21(1): 21.24 .

Singh, S., Beena, P.M. 2003. Profile of dermatophytes infections in Baroda. Indian J. Dermatol. Venerol. Leprol., 69:281-3

Surendran, K., Bhat, R.M., Boloor, R., Nandakishore, B., Sukumar, D. 2014. A clinical and mycological study of dermatophytic infections. Indian $J$. Dermatol., 59: 262-7.

Vikesh Kumar Bhatia and Prakash Chand Sharma. 2011. Epidemiological studies on Dermatophytosis in human patients in Himachal Pradesh, India, Springer Plus, 134 DOI: 10.1186/2193-1801-3-134.

\section{How to cite this article:}

Nabila Akbar and Kalyani, M. 2016. Clinico-Mycologicalal Profile of Dermatophyte Species in a Tertiary Care Center. Int.J.Curr.Microbiol.App.Sci. 5(11): 587-593.

doi: http://dx.doi.org/10.20546/ijcmas.2016.511.069 